\title{
Transarterial Chemo-embolization (TACE) prior to liver transplantation: Does it add to surgical risk when considering marginal grafts?
}

Authors: Amanda Carvalheiro, Carlos Derosas ,Yuri Boteon, Hynek Mergental, John Isaac, Paolo Muiesan ,Darius Mirza, Thamara Perera The Liver Unit, Queen Elizabeth Hospital Birmingham, United Kingdom

\section{Introduction:}

Loco-regional effects of TACE may adversely affect the technical aspects of LT, and hence implications for graft selection.

\section{Methods:}

Retrospective cohort study with HCC patients that underwent TACE before LT (TACE group) compared with patients that underwent LT for HCC but without preoperative TACE (NoTACE group), between January $201 \mathrm{I}$ and December 2015.

\section{Results:}

A total of 59 cases included in the TACE group were compared with 123 cases in the NoTACE group. TACE group had higher prevalence of ischaemia related native collateral damage (II.86\% vs. $0 \%$; $p=0.002$ ), native hepatic artery occlusion identified at surgery $(5.1 \%$ vs. $0 \% ; p=0.033)$ and adhesions between the necrotic tumour to adjacent structures $(14.75 \%$ vs. $3.15 \% ; p=0.005)$.Surgical morbidity rate was significantly higher in the TACE group considering Clavien-Dindo grade 2 complications $(64.4 \%$ vs $47.2 \% ; p=0.029)$, however this was not found for grade $\geq 3(45,8 \%$ vs. $58,5 \% ; p=0.105)$. Patients in the TACE group transplanted with grafts from donors after cardiac death had $8.7 \%$ 90-day mortality and $56.5 \%$ major complication rate compared to $6.7 \%$ and $65 \%$ for the NoTACE group $(p=0.719$ and $p=0.475$ respectively).

Table I: Postoperative mortality and morbidity

\begin{tabular}{|l|c|c|c|c|c|}
\hline & \multicolumn{2}{|c|}{ TACE 59} & \multicolumn{2}{c|}{ No TACE = I23 } & \\
\hline Variable & $\begin{array}{c}\mathrm{n} / \\
\text { median }\end{array}$ & $\% /$ IQR & $\begin{array}{c}\mathrm{n} / \\
\text { median }\end{array}$ & $\% /$ IQR & P-value \\
\hline \multicolumn{5}{|c|}{} \\
\hline Mortality & & & & & \\
\hline 30 days & 2 & 3.5 & 5 & 4.06 & 0.678 \\
\hline 90 days & 3 & 5.3 & 7 & 5.69 & 0.867 \\
\hline DBD $(\mathrm{n}=99)$ & 1 & 2.8 & 3 & 4.8 & 0.63 \\
\hline DCD $(\mathrm{n}=83)$ & 2 & 8.7 & 4 & 6.7 & 0.667 \\
\hline Overall morbidity & 50 & 84.7 & 100 & 81.3 & 0.568 \\
\hline DBD $(\mathrm{n}=99)$ & 29 & 80.6 & 49 & 77.8 & 0.745 \\
\hline DCD $(\mathrm{n}=83)$ & 21 & 91.3 & 5 & 85 & 0.719 \\
\hline $\begin{array}{l}\text { Morbidity, Dindo-Clavien } \\
\text { grades }\end{array}$ & & & & & \\
\hline I & 20 & 33.9 & 36 & 29.3 & 0.526 \\
\hline $\mathbf{2}$ & 38 & 64.4 & 58 & 47.2 & $\mathbf{0 . 0 2 9}$ \\
\hline 3 & 21 & 35.6 & 40 & 32.5 & 0.681 \\
\hline 4 & 15 & 25.4 & 47 & 38.2 & 0.09 \\
\hline Dindo-Clavien grade $\geq 3$ & 27 & 45.8 & 72 & 58.5 & 0.105 \\
\hline DBD $(\mathrm{n}=30)$ & 14 & 38.9 & 33 & 52.4 & 0.196 \\
\hline DCD $(\mathrm{n}=24)$ & 13 & 56.5 & 39 & 65 & 0.475 \\
\hline
\end{tabular}

$\S$ Some patients developed more than one complication. DBD, donor after brain death; DCD, donor after cardiac death

Table 2: Liver transplantation and postoperative data

\begin{tabular}{|l|c|c|c|c|c|}
\hline & \multicolumn{2}{|c|}{ TACE = 59 } & \multicolumn{2}{c|}{ No TACE = I23 } & \\
\hline Variable & $\begin{array}{c}\mathrm{n} / \\
\text { median }\end{array}$ & $\% / \mathrm{IQR}$ & $\begin{array}{c}\mathrm{n} / \\
\text { median }\end{array}$ & $\% / \mathrm{QQR}$ & $P$-value \\
\hline $\begin{array}{l}\text { Ischaemia related native } \\
\text { collateral damage }\end{array}$ & 7 & 11.86 & 0 & 0 & $\mathbf{0 . 0 0 2}$ \\
\hline $\begin{array}{c}\text { Adhesions between the } \\
\text { necrotic tumour to adjacent } \\
\text { structures }\end{array}$ & 9 & 14.75 & 4 & 3.15 & $\mathbf{0 . 0 0 5}$ \\
\hline $\begin{array}{c}\text { Native hepatic artery } \\
\text { thrombosis }\end{array}$ & 3 & 5.1 & 0 & 0 & $\mathbf{0 . 0 3 3}$ \\
\hline Native portal vein thrombosis & 4 & 6.8 & 18 & 14.6 & 0.151 \\
\hline
\end{tabular}

\section{Discussion:}

HCC patients undergoing TACE while in the LT waiting list may lead to complications related to the procedure experienced in the laparotomy, nevertheless it has not shown repercussions on the mortality and only partial impact at morbidity, even considering the use of marginal grafts.

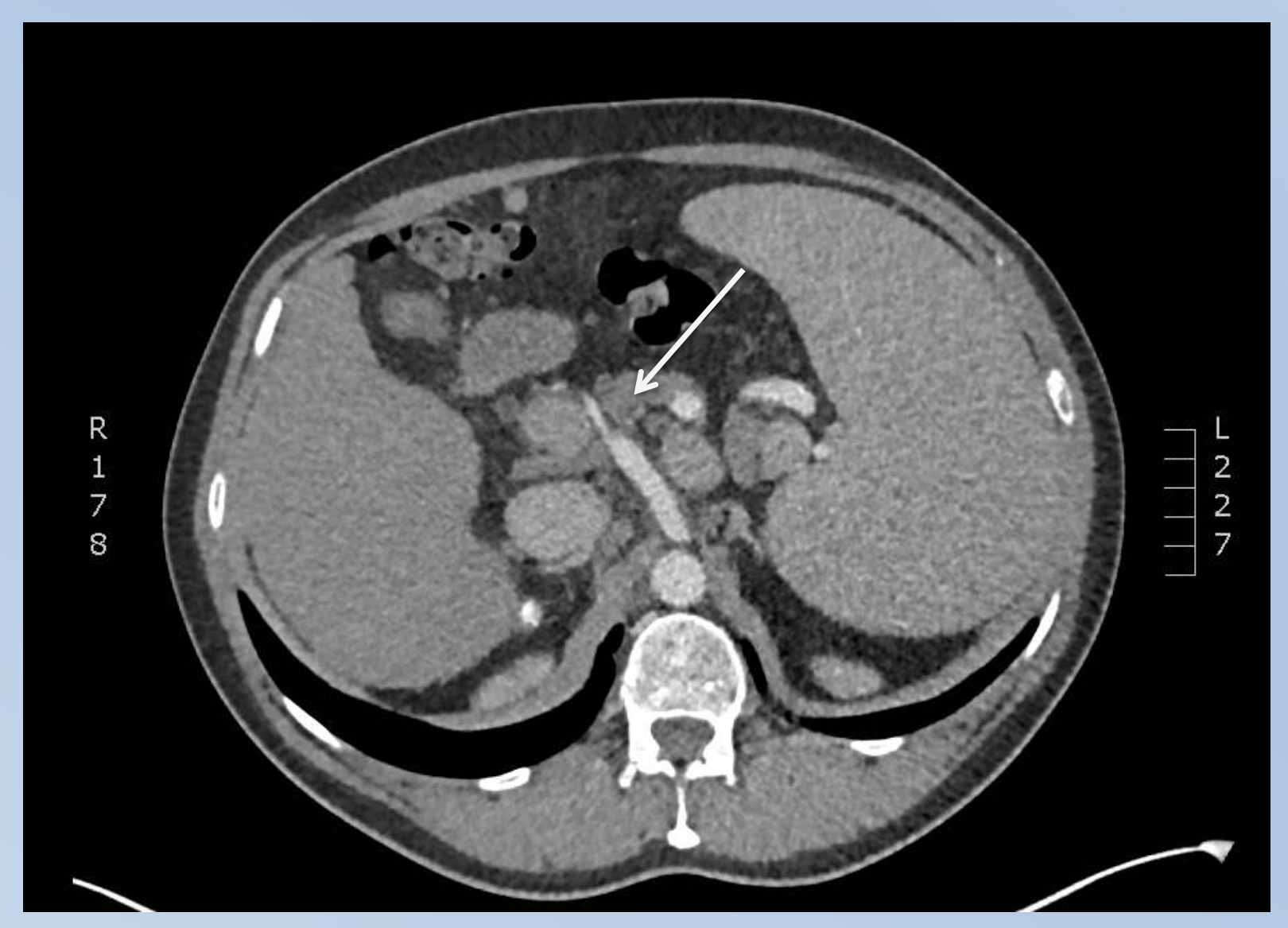

Figure I:CT post TACE shows narrowing CHA; Intra-operative findings: $\mathrm{CHA}$ with inadequate flow for the arterial reconstruction requiring aortic conduit.

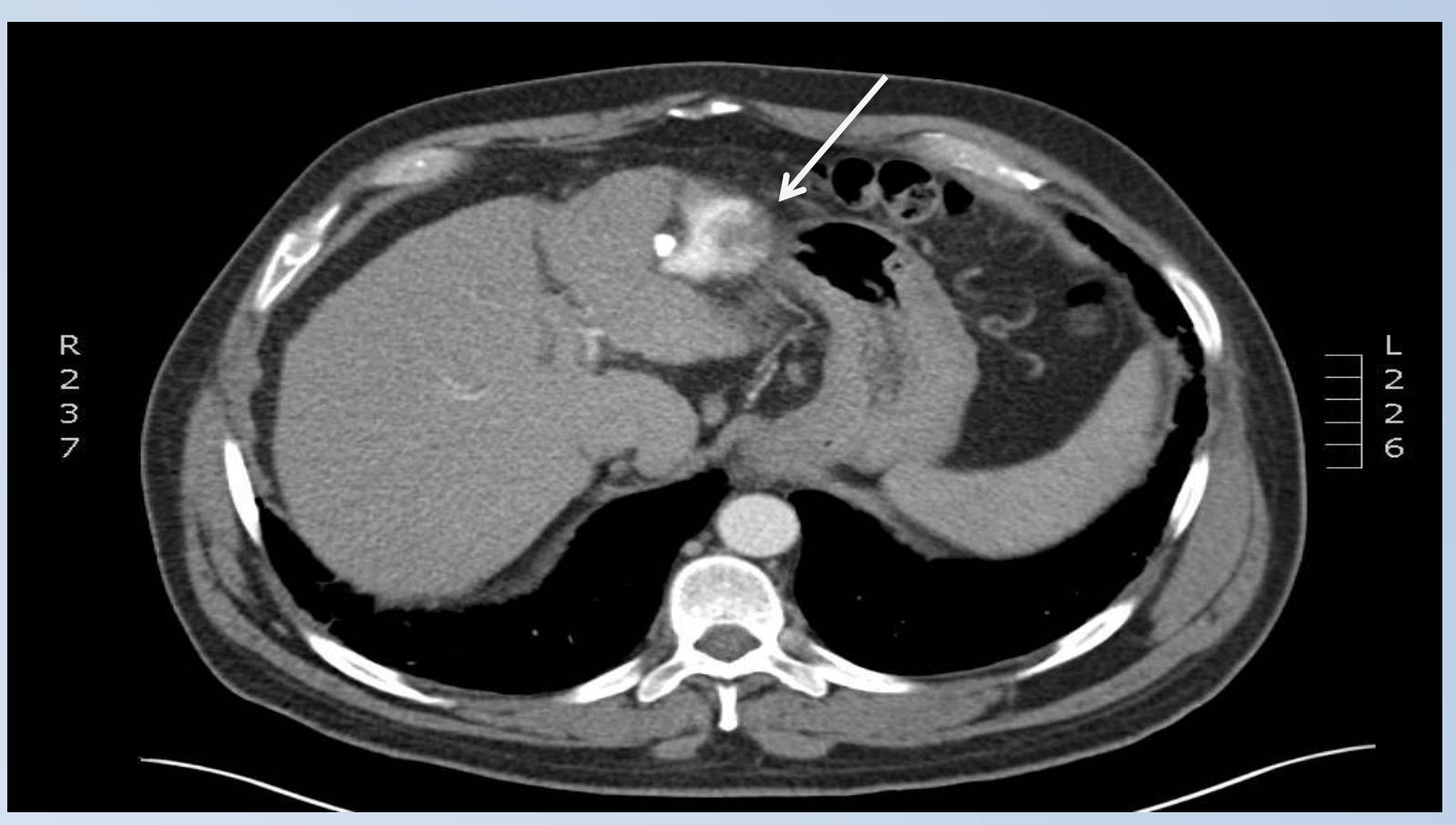

Figure 2:CT post TACE demonstrates tumour adherent to stomach, which was confirmed intra-operatively, requiring resection of a part of the anterior stomach wall.

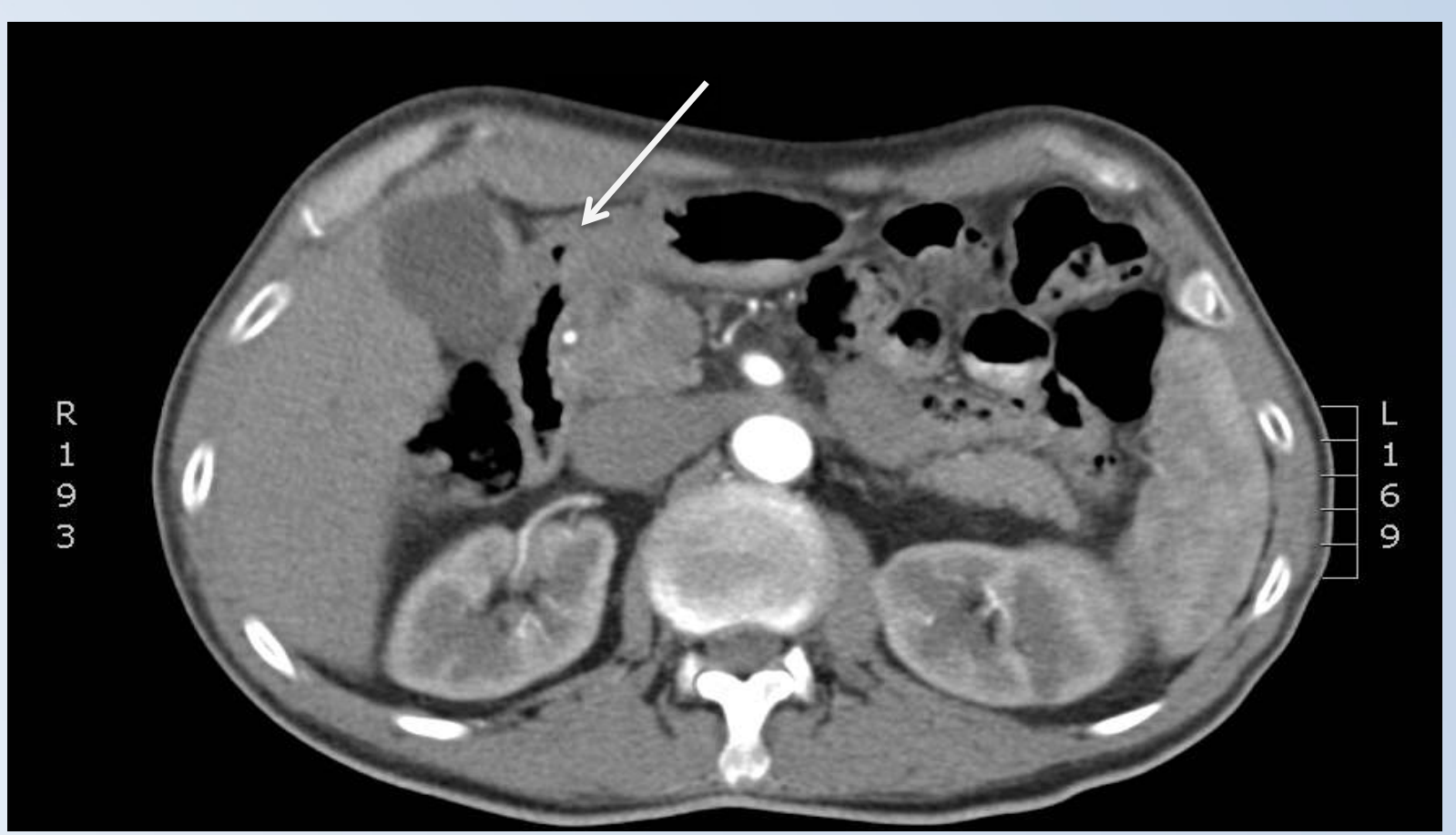

Figure 3:CT post TACE showed thickened gallbladder wall adherent to duodenum. This was confirmed intra-operatively associated to distorted anatomy in the hilum caused by inflammatory adhesions and duodenal repair was needed. 